\title{
Hybrid PET/CT and SPECT/CT Imaging
}

\author{
Thomas Leitha ${ }^{1}$ and Anton Staudenherz ${ }^{2}$ \\ ${ }^{1}$ Department of Nuclear Medicine, Danube Hospital, Vienna \\ ${ }^{2}$ University Clinic of Nuclear Medicine, Vienna \\ Austria
}

\section{Introduction}

\subsection{Definition of nuclear medicine, molecular imaging and hybrid imaging}

Nuclear Medicine uses radioactive probes commonly referred to as tracers for the diagnosis and treatment of diseases. Monitoring the metabolic fate of nanomolar amounts of radiolabeled substances by tracking their photon emission with SPECT (Single Photon Emission Tomography) and PET (Positron Emission Tomography) was the first widely practiced branch of Molecular Imaging (MI). MI is the visualization, characterization and measurement of biological processes at the molecular and cellular levels in humans and other living systems (Mankoff, 2007). Among the different techniques summarized in MI, tracer imaging in Nuclear Medicine has the highest molecular sensitivity, tracing substances in the $10 \mathrm{E}-3$ to $10 \mathrm{E}-5 \mathrm{~mol} / 1$ range. Conventional imaging with $\mathrm{x}$-ray was primarily used as a snapshot of anatomy and depicts tissue by its physical characteristics (e.g., X-ray density). Contrast agents in CT are used to increase the visibility of vessels and organ surfaces. Functional information is at best limited to that of perfusion and permeability. This is one of the most significant differences between Radiology and Nuclear Medicine.

The advantage of functional imaging is that of increasing sensitivity because metabolic changes precede anatomical changes and can be detected long before structural changes appear. Functional imaging, however, has a low specificity in distinctly different pathologies (e.g., degenerative, inflammatory or malignant bone lesions) if they are visualized by unspecific common properties as hyperaemia, increased regional tracer permeability and osteoplastic metabolism. To overcome this and to increase specificity, highly specific probes (e.g., receptor imaging) have been developed. With minimal uptake outside the targeted tissues, specific probes offer little information about the surrounding tissues and consequently do not provide the topographical information needed by surgeons or therapy planning systems in radio oncology. The advantage of anatomical imaging by CT is its high anatomical resolution and usually good topographical information. On the other hand it is a poor predictor of the functional consequences of a finding (e.g., borderline stenosis in coronary CT angiography) and consequently a poor predictor of prognosis. Structural data do not necessarily correlate with the metabolic status of disease and they have a limited diagnostic sensitivity in cases with abnormal anatomy (e.g., scar versus residual tumour). Anatomic tumour response metrics (WHO criteria, Response Evaluation Criteria in Solid Tumours (RECIST)) are insufficient to predict therapy response, particularly in assessing the activity of newer cancer therapies that stabilize disease and may 
be substituted by quantitative approaches in functional imaging (PET Response Criteria in Solid Tumours; PERCIST (Wahl et al 2009)). An ideal non-invasive technique provides complementary information on topography and anatomy as well as on the functional behaviour of a lesion.

Less than one decade ago, primarily forced by the manufacturers, hybrid devices were brought into clinical routine. It took only a few years after the first description of a working prototype (Beyer et al., 2000) to replace stand-alone PET by PET/CT. A similar success story may be appreciated with SPECT/CT (Schillaci et al., 2005). However, the development of hybrid imaging in PET and SPECT followed different pathways. Whereas PET was always combined with a state-of-the-art CT system, the first SPECT/CT design was based on a lowcurrent, slow revolving CT (see chapter 2). Both hybrid devices have been shown to improve diagnosis and risk stratification by combining anatomical and functional information (see chapters 4 and 5).

Nuclear Medicine interpretation benefits from CT in three ways:

Firstly, scintigraphic uptake can now be localized topographically and enables local interventions (e.g., surgery, radio therapy planning). Secondly, physiological and unphysiological tracer uptake can be better discriminated by visualizing the anatomical background (e.g. FDG uptake in brown adipose tissue). Thirdly, an often overlooked advantage is that the $\mathrm{CT}$ attenuation map shortens imaging protocols and possibly improves attenuation and scatter correction of PET and SPECT.

On the other hand, Nuclear Medicine may also improve CT interpretation, e.g. in the differential diagnosis of minimally enlarged mediastinal or retroperitoneal lymph nodes and in therapy monitoring.

\section{$\mathbf{1 . 2}$ Is hybrid imaging the final answer to all our needs?}

The complementary information of anatomical and functional imaging has been used for decades to define, characterize, stage and monitor disease, but until recently the different modalities have been performed in sequence (e.g. screening for bone metastases by whole body bone scanning followed by planar radiographs and possibly MRI). Before hybrid systems (PET/CT, SPECT/CT) came into the market the findings of different modalities had to be independently viewed or (as discussed in chapter 3.) digitally fused. The coming of hybrid devices was welcomed by many with overwhelming optimism (von Schulthess et al., 2002), but a more thorough assessment of the matter surfaces several unsolved problems which will be addressed below. Sceptics may even say that we - driven by the market and our own curiosity - have opened Pandora's box.

\subsection{What is the incremental benefit of simultaneous hybrid imaging to the sequential use of optimized imaging?}

The most obvious answer to this question seems to be that patient positioning and movement is not an issue in simultaneous hybrid imaging but sime of these problems may have not been eliminated. Modern systems combine both modalities on a single gantry, but different detectors are still needed to image both signals and both studies are acquired sequentially. The problems of misalignment of both investigations are discussed in detail in 1.6.1. The strongest evidence for the clinical effectiveness of FDG-PET is in staging NSCLC, restaging HL, staging/restaging colorectal cancer and detection of solitary pulmonary nodule (Facey et al., 2007). Numerous studies with a heterogeneous design claimed that the 
incremental benefit of simultaneous hybrid imaging to sequential protocols is well over 10 $\%$ at least in oncology (Czernin et al., 2010, Delbecke et al., 2009).

\subsection{Can the additional accuracy be utilized in clinical practice?}

The cost-effectiveness of a PET plus CT strategy was shown as early as in the late 90s (Gambhir et al., 1996) for diagnosis and staging of lung cancer and numerous other indications followed. Nevertheless especially in follow-up investigations, it all comes down to this:

Does the additional information affect clinical decisions in a significant number of patients and do they benefit from the additional information provided by advanced imaging?

There is some evidence in oncology that hybrid imaging has changed patient management (Almuhaideb et al. 2001, Facey et al., 2007). In cardiology the addition of calcium scoring has been described to improve the results of myocardial perfusion SPECT (Mahmarian, 2007). Though sometimes quoted (Farid et al., 2009) data are scarce that hybrid imaging in neurology has an additional advantage to digital image fusion.

\subsection{Is the additional radiation burden of multiple diagnostic CTs worthwhile?}

In Nuclear Medicine imaging can be performed as whole body imaging or dual phase protocols without additional radiation exposure. In contrast, multi-phase CT protocols and larger scanning volumes inevitably increase the radiation burden of the patient. Routinely combining PET or SPECT with diagnostic whole body CT unnecessarily increases the

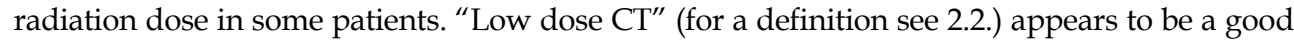
compromise, but it may overlook minute pathologies that show no tracer uptake but could have been revealed by a diagnostic CT. Diagnostic CTs in radiology (in contrast to low-dose CTs in Nuclear Medicine) are routinely acquired during maximum inspiration, increasing the intrathoracic volume, moving the diaphragm downwards and changing the relative organ positions in comparison to the situation during the PET/SPECT acquisition. A mid expiratory position would be a good compromise but is difficult to reproduce voluntarily (Pan et al., 2005) and it has yet to be determined to what extent this hampers the diagnostic accuracy of the CT in the lung. A step wise approach should be ideal but this is very difficult to implement into the busy scheduling of diagnostic imaging when all too often the tentative diagnosis of the referrer is imprecise and it is hard to decide the necessary depth of the diagnostic protocol. Thus, at present many CTs will be duplicated in hybrid imaging.

\subsection{Is the CT information fully utilized in the reconstruction of PET and SPECT imaging?}

Until recently most reviews about hybrid imaging have focused on the clinical advantages due to the better anatomical allocation of PET/SPECT images, but at least in PET/CT the biggest advantage was that of improving image reconstruction by speeding the transmission scan. Coincidence imaging in PET and Single Photon imaging in SPECT imaging, though completely different in some of their physical basics, need several corrections to improve imaging and to allow quantification, with attenuation and scatter correction being the most important. Whereas these corrections are now regarded as mandatory in PET, they may gain importance in quantitative SPECT, but at present are only implemented in myocardial perfusion imaging and brain imaging. Initially both, PET and SPECT, used radioactive sources for transmission imaging, but attenuation maps generated from CT scans can be 
acquired much faster. However, three issues may hamper this approach: Misalignment, energy dependent attenuation and CT artefacts.

\subsubsection{Misalignment}

Misalignment of both studies cannot be avoided completely as different detectors are needed to image signals from radionuclides and x-ray tubes (Goetze \& Wahl, 2007). Both studies are acquired sequentially and differ significantly in imaging duration thus causing head, whole-body and extremity motion, diaphragmatic motion with breathing and bowel motility (Figure 1). The different expiratory positions between CT and PET/SPECT have been addressed in 1.5.

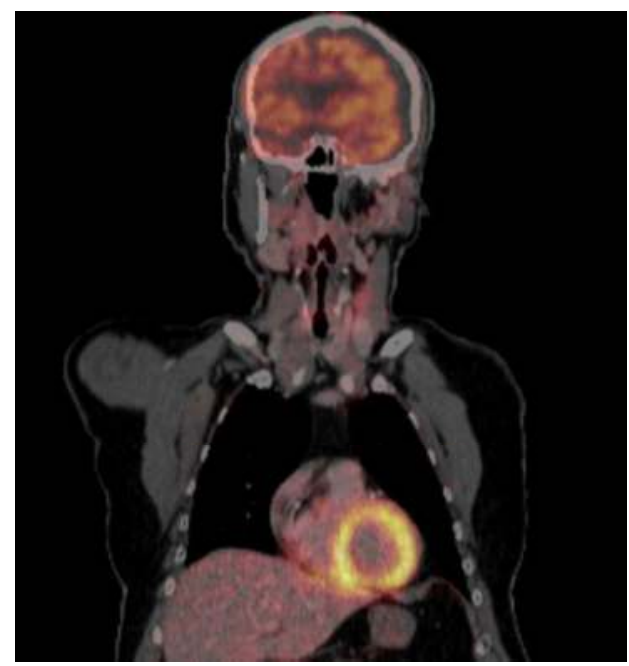

Fig. 1. FDG PET/CT with minimal head movement between both investigations

\subsubsection{Energy dependent attenuation}

The different energy spectrums of Nuclear Medicine tracers (PET monoenergetic 511-keV photons, SPECT mono- and multienergetic photons in a wide range of energies) and CT pose another challenge. The spectrum of photons energy from the anode of the $x$-ray tubes used in CT range from $0 \mathrm{keV}$ up to peak energy used for the acquisition. Attenuation increases with lower energy this may cause artefacts, like beam-hardening effects. So, filtering of the beam is required. The resulting spectrum has a mean energy of about $70 \mathrm{keV}$. At the low energy level of some SPECT tracers (e.g., 68-80 keV (94.5\%) Tl-201) and CT photons, interaction with body tissues takes place in part through photoelectric effects, whereas the high energy 511-keV annihilation photons used for PET are almost exclusively attenuated by Compton scattering. The attenuation coefficients need to be scaled appropriately in each energy window for the different materials (air, soft tissue, bone). This is more complex for SPECT than for PET, due to the wide range and sometimes multiplicity of energy windows of the used radioisotope. Before CT data can be used for attenuation correction in PET and SPECT, CT voxel sizes have to be downscaled due to the lower spatial resolution of those devices (Krishnasetty et al., 2008) and segmented according to the different materials. The used algorithms differ between different vendors and apparently 
have been set for optimized visual diagnosis and not quantitative requirements. The consequences for quantitative PET/CT have been summarized elsewhere (Weber et al., 2007). Until now quantitative SPECT was not a major issue but is rapidly emerging for diagnostic (fp-cit scan) imaging and dosimetry (Patton \& Turkington, 2008).

\subsubsection{CT artefacts}

Attenuation artefacts (dental work, metallic implants) are other possible problems if CT is used for correction purposes in Nuclear Medicine imaging. Routine reconstruction for CT is filtered back projection and not iterative reconstruction and the first is especially susceptible to beam hardening artefacts if the patient does not fit into the field of view. This issue becomes more evident if low dose parameters are used and artefacts may hamper PET and SPECT reconstruction (Figure 2).

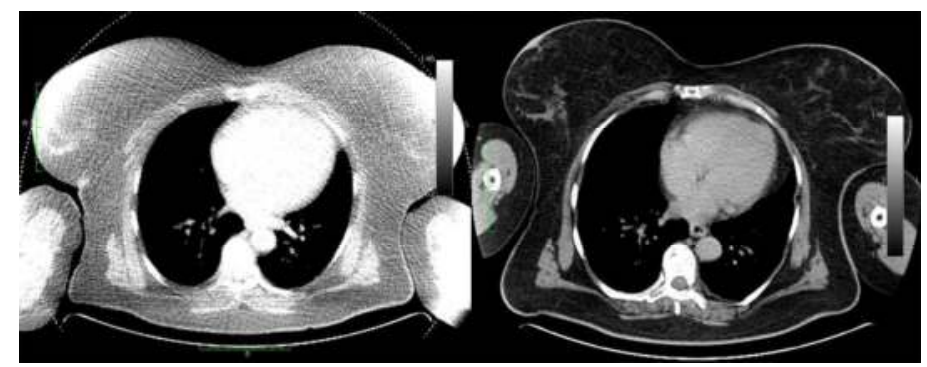

Fig. 2. Beam hardening artefacts in a large patient (height: $175 \mathrm{~cm}$, body weight: $120 \mathrm{~kg}$ ) at the edge of the field of view are evident in the image of the low dose CT $(140 \mathrm{kV}, 230 \mathrm{mAs})$ on the left and have disappeared in the image on the right after conventional CT parameters $(140 \mathrm{kV}, 300 \mathrm{mAs})$ have been applied.

This problem might be partially solved by using iterative reconstruction algorithm (Kinahan et al., 2003), but things get even more complicated if contrast media are involved in hybrid imaging (Büther et al., 2007). The different energy spectra of SPECT tracers pose an additional challenge to the reconstruction process if absolute quantification is aspired.

\subsection{Do we need hybrid imaging in all situations?}

Most likely not, but a more detailed analysis reveals a complex picture. Critics apparently have ample evidence of accusing our professions to succumb to a "the more the merrier" principle. We definitely have to provide evidence in which clinical situations hybrid imaging is warranted but then little is gained if a given diagnostic tool has shown to be the method of first choice in a certain situation, if this tool is not accessible: In most areas patient access to CT is much easier than to PET/CT or SPECT/CT thus many patients will already have a diagnostic CT before coming to Nuclear medicine imaging. This opens up the field for digital image fusion as described below (Chapter 3.).

\section{From low dose to multi-slice CT}

\subsection{History and milestones}

The origins of PET, SPECT and CT date back into the early 1970s. Burnham et al. (1972) developed a multi-crystal positron camera. Modern PET scanners are based on the work of 
Phelps et al (1975), Ter-Pogossian et al. (1975) and Hoffmann et al (1976). Almost in parallel in the early 1970s Ambrose \& Hounsfield (1973) introduced a computerized x-ray tubebased tomographic scanner providing images of tissue densities from acquired projections. In contrast to the evolution of SPECT/CT the first PET/CT scanner was developed as a combination of a then state-of-the-art spiral CT (Siemens Somatom AR) and a partial-slip-ring "poor man's PET" (ECAT ART PET) on a common rotational support within a single gantry with the PET components on the reverse side of the rotating support of the CT scanner. This design reduced the expensive detectors from 144 to 66 and brought the price tag down to below 1 Million US \$. Townsend et al., (1998) published their combination SMART scanner providing sequential imaging using the table as shuttle using the advantage of having the patient in the same body position without changing. Almost a decade later the first prototype SPECT/CT system was developed (Lang et al., 1991). This prototype used the same high-purity germanium detector array for both x-ray and single-photon imaging. The same group (Hasegawa et al., 1993) developed a different design by combining a CT scanner with a commercial gamma camera but it was not until the millennium when a different design became the first commercially available SPECT/CT system. This was in part triggered by the fact that this manufacturer (then ELSCINT, now General Electrics) was the first who used a slip ring for its gamma camera gantry, allowing $>360^{\circ}$ rotation. This design was based on low speed. low current CT (Hawkeye) that was explicitly developed for hybrid imaging with the Infinia gamma camera. At this time this was the logical approach as it provided sufficient topographic orientation and a transmission map for attenuation and scatter correction closely resembling the organ positions during the SPECT acquisition. The Hawkeye opened the market for SPECT-CT, but surprisingly or not in the end the market voted for other designs with MD CT scanners (Siemens, Philips). Siemens introduced the Symbia series in 2004 followed by Philips Precidence. Currently available SPECT/CT systems are: Discovery NM/CT 670 (General Electric), BrightView X/CT (Philips) and the Symbia T series (Siemens). Today PET/CT systems are only available with diagnostic CT scanners. The CT system can be operated at reduced tube current if only used for attenuation correction. The latest development in the history of PET/CT appears to be the marketing of a "CT/PET". Siemens hides the fact that they are selling a PET/CT by naming it "molecular CT" (Biograph mCT). In 2008, the first hybrid PET/MRI system for humans was installed by Siemens (Pichler et al., 2008); but that is a completely different story.

\subsection{Radiation exposure in hybrid imaging}

Radionuclides used in PET and SPECT have a much lower photon flux and higher $\gamma$-rays energy in comparison to x-rays in CT. The effective dose caused by intravenously applied tracers in Nuclear Medicine is not only affected by the physical properties of the radionuclide but even more so by the biokinetics of the radiopharmaceuticals, thus in i.v. studies all organs are exposed to some extent. In contrast, the absorbed dose in CT is dependent on several operator dependent factors (mAs (directly proportional to radiation dose), $\mathrm{kVp}$ (not linearly proportional to dose), pitch (inversely related to dosage), slice thickness (smaller slices increase mAs), number of scans (multiplying radiation dose), scan time (faster scans lead to an increased scan area), scanning mode (step-based, spiral technique; single-slice, multi-slice) and proportional to the absorption coefficient of the irradiated tissue that is more or less limited to scanned body volume) (Bauhs et al., 2008). 
Initially, attenuation correction for PET and SPECT was based on transmission scanning with radioactive sources (153-Gd for SPECT, 68-Ge for PET, respectively). Those sources exposed the patient to only low additional effective doses in the order of 0.1 to $0.2 \mathrm{mSv}$ (Delbeke \& Israel, 2010). Using CT as transmission source, especially in a diagnostic setting, the effective dose rises up to 100 times depending on the imaging protocols. A diagnostic CT of the chest, abdomen and pelvis will give an effective dose of about $13 \mathrm{mSv}(140 \mathrm{kV}, 340$ mAs, $0,5 \mathrm{~s}$ rotation time and a pitch fo $17.5 \mathrm{~cm} / \mathrm{s}$ ). On the other hand 18-F FDG PET (370 $\mathrm{MBq}$ ) causes an effective dose of about $11 \mathrm{mSv}$. The effective dose of SPECT varies, due to a wide range of different tracers. $99 \mathrm{~m}-\mathrm{Tc}(925 \mathrm{MBq})$ labelled white blood cell imaging is an investigation with one of the highest effective doses and delivers $18.5 \mathrm{mSv}$ (Stabin et al., 1996) to the patient. In commonly applied protocols in Austria (Stemberger et al., 2011) the contribution of the CT to the average total body effective dose is $14 \mathrm{mSv}$ in PET/CT and 1-3 $\mathrm{mSv}$ in SPECT/CT. Moving from diagnostic to "low dose" CT protocols lowers the effective dose considerably. Low dose CT is said to make up for only $10 \%$ to $15 \%$ of total effective dose in hybrid imaging, but some caution is advised: There is considerable confusion in the definition of the term "low dose" CT in hybrid imaging. Referring to the definition of the American College of Radiology (ACR) for Relative Radiation Level (RRL) values (Table 1), very few of the proposed protocols qualify for the definition of low radiation level.

\begin{tabular}{|c|c|}
\hline Radiation Level & Effective Dose Range \\
\hline None & 0 \\
\hline Minimal & $<0.1 \mathrm{mSv}$ \\
\hline Low & $0.1-1 \mathrm{mSv}$ \\
\hline Medium & $1-10 \mathrm{mSv}$ \\
\hline High & $>10 \mathrm{mSv}$ \\
\hline
\end{tabular}

Table 1. Modified according to the ACR Appropriateness Criteria

To put this into a clinical perspective, one has to address the issue how to quantify radiation risk: Basically, the effective dose does not reflect the individual risk but should be used to compare systems and to justify and optimize procedures. The individual risk is estimated by calculating mean doses of all organs and tissues in relation to age, sex and organ specific risk coefficients (ICRP 2007). The primary risk associated with radiation exposure in diagnostic imaging is the risk to develop cancer and this appears to be comparatively high. The seventh National Academy of Science report on Biological Effects of Ionizing Radiation estimated that a single dose of $10 \mathrm{mSv}$ produces a lifetime risk of developing a solid cancer or leukemia of 1 in 1000 (BEIR VII 2006). This, however, has to be appreciated in the light of the chance of 1 in 4 dying from cancer in the developed countries. The most important way to reduce radiation dose in hybrid systems is to optimize the CT protocols. Most companies recommend protocols for optimal visual image quality but the question remains to what extent CT parameters can be modified to reduce dose without losing adequate information for attenuation correction, quantification and diagnostic information. Neuwirth et al., (2010) using a Siemens Biograph 64 PET/CT scanner showed that the attenuation correction of PET is not hampered even if the lowest possible CT parameters $(80 \mathrm{keV}, 28 \mathrm{mAs})$ are used. It goes without saying that those CT images contribute little diagnostic information. At present we lack sufficient data to quantify the diagnostic trade off between "low dose" and "diagnostic" CT. To this point we have only discussed the contribution of the x-ray tube and 
radiopharmaceutical to the effective dose of the patient. Often neglected is the contribution of the scout scan or topogram used to determine scan limits before acquisition starts to total radiation exposition. A recent study (Moore et al., 2010) showed that the additional dose contributed by the topogram can be minimized ( $25 \mu \mathrm{Gy})$ but in clinical routine it may be as high as 10 to $20 \%$ of the diagnostic CT scan depending on exposure time, region length, $\mathrm{mA}$ and voltage (O'Daniel et al., 2005). In principle, the topogram could be easily substituted in hybrid systems by the emission data of PET or SPECT but this option is still not available in all current hybrid designs as most are basically two devices glued together working independently.

\section{From digital Image fusion to hybrid imaging}

Before the advent of hybrid devices several steps had to be taken to allow digital image fusion of CT and Nuclear Medicine techniques. Whereas Nuclear Medicine imaging became digital very early in its development, digital radiology emerged not before the late $80 \mathrm{~s}$. The second requirement was an interchangeable image format for both modalities. Initially images in Nuclear Medicine were stored in company proprietary formats. With several intermediate steps (e.g. interfile), the current definition of the DICOM standard (http://medical.nema.org/) allows handling, storing, printing, and transmitting of most study types in medical imaging. Nowadays accepted as natural law (Moore's law), the gigantic boost in computer power was mandatory to implement sophisticated iterative reconstruction and fusion algorithms in clinical routine. Often overlooked, but sufficient in some clinical situations, even simple manual fusion has an incremental diagnostic impact over the separate analysis (Nakamoto et al., 2008). However, if image-guided interventions or sophisticated dosimetry is needed, digital fusion is a necessity. Numerous algorithms have been described to retrospectively align 3-dimensional data acquired by stand-alone modalities to common spatial coordinates (Slomka, 2004). Two basic approaches (internal landmarks or external fiducials) can and have been used to fuse tomographic images (Kramer et al., 1991; Kessler, 2006). Whereas external markers with individualized masks are routine procedures in radio oncology, they require considerable logistics, can only be used for small volumes and neglect information about internal organ displacements. Internal landmarks have the advantage of correcting for different organ positions and can be used even if the first study was acquired without the knowledge of a later image fusion but require substantial user interference which is prone to errors and may lack reproducibility. Basically all the used algorithms can be separated into feature or volume based approaches. The first align the two image sets according to extracted image features (e.g. anatomic landmarks, organ surfaces ...) the latter maximize measures of similarity between images. Another important difference between these algorithms is if they are linear or nonlinear. Linear image fusion subjects all coordinates to the same extent of translation, rotation and scaling to be fused with the second study. This is perfect for the brain but insufficient for the non-rigid complex geometry of thoracic volumes. In contrast, nonlinear techniques are capable of fusing different intrathoracic volumes and to some extent different patient positioning but fail to correct for internal organ displacement, namely the liver. It has been reported that integrated PET/CT devices provided additional information in approximately $6-7 \%$ of all lesions. However, in this report (Reinarzt et al., 2004) abnormal CT findings in the absence of increased 18F-FDG uptake were excluded, thus have not strictly investigated the additional benefit of the combined devices. Available evidence for the incremental 
diagnostic utility of hybrid imaging in comparison to visual or digital fusion will be discussed below, but it is safe to predict that digital image fusion will continue to play an important role in patients with a previously performed diagnostic CT or MR.

\section{Current state of clinical PET/CT}

A recent survey showed PET is most frequently used in oncology (87\%) followed by neurology $(5 \%)$, radiation therapy planning and cardiology $(4 \%)$, respectively. I.v. or oral contrast was used at $52 \%$ of the sites in up to $25 \%$ of patients but only $62 \%$ of the sites provided a fully integrated PET/CT report (Beyer et al., 2011). As stand alone PET systems have disappeared the clinical question is not if $\mathrm{CT}$ is used but to what extent (dose, contrast enhancement) it is performed as a diagnostic CT (Coleman et al., 2005).

\subsection{Oncology}

The utility of FDG PET in oncology has been recently summarized (Facey et al., 2007, Fletcher et al., 2008). PET/CT systems were not explicitly addressed due to the paucity of published data. Data for other PET tracers are not summarized here because of an even more limited database. Despite being the standard of care, there has been uncertainty about whether or not improved diagnostic accuracy translates into improved management of patients. The strongest evidence for the cost-effectiveness of PET is still in the staging of non-small cell lung cancer, but a recently performed analysis of FDG PET in oncology found only four studies (Mansueto et al., 2009, Lejeune et al., 2005, Krug et al., 2010, Yen et al., 2009) that based their findings explicitly on PET/CT (Langer, 2010). The following overview tries to suggest clinical setting where growing evidence exists that the initial use of a diagnostic CT or if no access to hybrid imaging is possible, image fusion between CT and PET has to be aspired.

\subsubsection{Head neck cancer}

Accurate $\mathrm{T}$ and $\mathrm{N}$ staging requires contrast enhanced $\mathrm{CT}$ and PET and both investigations are complementary. Reactive lymph nodes in CT can be identified if FDG uptake is very low and necrotic lymph node metastases can be diagnosed in CT that are false negative in PET due to a low FDG uptake (Schöder et al., 2004, Jeong et al., 2004). However, as $40 \%$ of cervical metastases are below one centimeter in diameter not even PET/CT can confirm N0 necks thus opening up the field for sentinel imaging (Hyde et al., 2003), possibly performed as SPECT/CT (see 5.1.3). Therapy monitoring should not be performed earlier than one month after therapy, due to a $>30 \%$ rate of false negatives (Rogers et al., 2004, Engles et al., 2006). Restaging with FDG PET is more sensitive than CT/MR (Klabbers et al., 2003) but we lack data if PET/CT could further improve diagnosis.

\subsubsection{Lung cancer}

The sensitivity of integrated FDG PET/CT for mediastinal lymph node staging is low. $\mathrm{PET} / \mathrm{CT}$ is superior to PET or CT alone, and visual correlation of both techniques separately. In particular, it improves T3 and T4 staging and delineation of tumours associated with atelectasis (Gámez et al., 2006). When positive mediastinal lymph nodes are detected, invasive mediastinal staging has been advised. On the other hand, the specificity is high: patients with negative integrated FDG PET-CT can be operated upon without invasive 
mediastinal staging (Perigauda et al., 2009). Evidence accumulates that in patients with locally advanced NSCLC, quantitative analysis of a routine whole-body FDG PET/CT studies predicts tumour response to therapy (Eschmann et al., 2007).

\subsubsection{Esophageal cancer}

Endoscopic ultrasound (EUS) is superior to PET/CT for $\mathrm{T}$ staging and in identifying locoregional nodes but PET/CT has a high sensitivity and specificity for M staging. EUS and PET/CT independently affects treatment decisions, indicating complimentary and necessary roles in the staging (Walker et al., 2011). PET/CT is not sufficiently reliable in the individual patient for monitoring of treatment response but effective in detecting recurrent disease (Weber et al., 2001, Munden et al., 2006, Bruzzi et al., 2007, Kim et al., 2009).

\subsubsection{Colorectal cancer}

PET/CT is recognised in the preoperative evaluation of apparently limited metastatic disease, detection of disease recurrence, clarification of equivocal lesions, investigation of rising tumour markers, and incidental detection of occult primary colonic tumours (Chowdhury et al., 2010). Transrectal ultrasound and MRI provide much better anatomic resolution and are of greater value for $\mathrm{T}$ staging. The sensitivity for regional $\mathrm{N}$ staging is low (Kantorva et al., 2003). Yet, in a study of patients with low rectal cancers, FDGPET/CT altered treatment plans in 38\% of patients largely through the detection of unsuspected inguinal adenopathy (Gearhart et al., 2006). PET for diagnosis and staging does not generate additional survival effectiveness compared with CT alone but cost savings associated with its use and the improvement of therapeutic management (Lejeune et al., 2005).

\subsubsection{Hepatic metastases}

Size is a limiting factor and the additional value of PET/CT is not yet fully established (Wiering et al., 2008).

\subsubsection{Breast cancer}

The present evidence does not support the routine use of PET or PET-CT for the assessment of the clinically negative axilla but is superior to conventional staging for detecting internal mammary chain nodes (Cooper et al., 2011, Segaert et al., 2010). FDG-PET/CT changes the clinical management in $50 \%$ of breast cancer patients with elevated tumour markers and negative or equivocal conventional imaging modalities (Filippi et al., 2011). Several studies aimed to use PET/CT to monitor therapy response, yet lack sufficient validation (Groheux et al., 2011). PET/CT showed clear advantage over CT and PET alone for the diagnosis of breast cancer recurrence (Pennant et al., 2010).

\subsubsection{Melanoma}

The combination of PET and CT had a higher sensitivity than either technique alone in staging high risk-patients with potentially respectable cancer (Strobel et al., 2007). Integrating PET-CT in the management of patients with high risk MM appears to be less costly and more accurate by avoiding futile thoracotomies in one of five patients as well as by providing a small survival benefit at 10 years (Krug et al., 2010). 


\subsubsection{Lymphoma}

PET/CT improves baseline staging compared with conventional staging with CT alone (Freudenberg et al., 2004) and has been extensively reviewed in this field (Cronin et al., 2010). PET/CT scanning had a significant predictive value for disease progression and survival of DLBCL in post-rituximab treatment; it might be the single most important determinant of clinical outcome in patients with the same IPI risk (Yang et al., 2011). Nevertheless it is still not proven that the use of interim FDG PET can improve patient outcomes (Hutchings et al., 2009).

\subsubsection{Ovarian cancer}

FDG PET/CT is not recommended for primary ovarian cancer detection but is the best technique for lesion detection and treatment follow-up (Veena et al., 2010). It alters management in close to $60 \%$ of patients, detects more sites of disease than abdominal and pelvic CT and is superior in the detection of nodal, peritoneal and subcapsular liver disease (Fulham et al., 2009).

\subsubsection{Gastrointestinal stromal tumors (GIST)}

PET/CT is the method of choice for staging of GIST because some lesions have only a weak FDG uptake. PET/CT was also successfully used for monitoring of imatinib therapy because tumour shrinkage is minimal even in histological successful therapy (Antoch et al., 2004).

\subsubsection{Radiotherapy planning}

Nine experts and three IAEA staff evaluated the use of PET in radiotherapy planning and considered integrated PET/CT as the best available approach in non-small cell lung cancer, head and neck cancers, lymphoma and in esophageal cancers, with promising preliminary data in many other cancers (MacManus et al., 2009, Paulino et al., 2003).

\subsubsection{Thyroid cancer}

FDG PET/CT is capable to detect disease in patients with differentiated thyroid carcinoma with elevated $\mathrm{Tg}$ levels and negative radioiodine scan and the localization of disease in patients with medullary thyroid cancer and elevated serum calcitonin levels (Basu et al., 2011). 124 Iodine PET/CT may serve a role in obtaining lesional dosimetry of I-131 therapy (Abraham \& Schöder, 2011).

\subsubsection{Prostate cancer}

The role of PET in prostate cancer has not been established yet, but due to the limited anatomical information provided by the used tracers, notably radiolabeled choline, PET/CT will be mandatory for local therapy planning.

\subsection{Neurology}

We lack evidence that attenuation and scatter correction in the brain is significantly improved by CT in contrast to conventional correction. It might however be useful for image landmarking in image fusion with MR.

\subsection{Cardiology}

Although the introduction of hybrid PET/CT technology offers the exciting possibility of assessing the extent of anatomic CAD (CT coronary angiography) and its functional 
consequences (ischemic burden) in the same setting, there are technical challenges in the implementation of CT-based transmission imaging for attenuation correction (DiCarli et al., 2007). The clinical potential of PET/CT for the characterization of cardiovascular diseases has been reviewed recently (Schwaiger et al., 2010) and is apparently used in clinical routine with increasing frequency (Heller et al., 2009).

\section{Current state of clinical SPECT/CT}

Whereas the PET market has entirely focused on hybrid designs, it is still possible to buy stand alone SPECT systems, but in view of the commercial success of integrated PET/CT scanners, there is an increasing interest in SPECT/CT systems (Buck et al., 2008). World wide market sales according to an industry source are in the range of $75 \%$ SPECT and $25 \%$ SPECT/CT with a continuing increase of hybrid devices.

\subsection{Oncology}

The role of hybrid imaging in oncology has been recently reviewed (Chowdhury \& Scarsbrook, 2008).

\subsubsection{1-I thyroid cancer}

Planar 131-I whole body scan is hampered by many pitfalls (Leitha \& Staudenherz, 2003). SPECT/CT improves the differentiation between physiologic and pathologic uptake of whole body iodine scan (Kohlfürst et al. 2009) and changes the therapeutic approach in a significant group of patients (Yamamoto et al., 2003). The unique contribution of a diagnostic CT in addition to the information of the 131-I scan is the detection of non-iodineavid nodular lung disease, lymph node metastases and lytic bone lesions. It remains to be determined to what extent these findings improve patient management.

\subsubsection{Neuroendocrine tumors, neural crest tumors}

Low dose CT improves image interpretation by providing a better anatomic localization of SPECT-detected lesions in 41 percent of the patients primarily by improving the discrimination between physiological and pathological tracer uptake (Even-Sapir et al., 2001). Pfannenberg et al., (2003) pointed out that "The problem of false-negative SPECT results cannot be solved by SPECT/CT because of inherent low resolution and lack of oral and intravenous contrast of the low-dose CT component. SPECT/CT is better than SPECT or CT alone but cannot replace high-end CT. Therefore a high end CT component should be implemented in the SPECT/CT device to allow for combined acquisition of high-quality contrast-enhanced CT in addition to SPECT." I-123 mIBG SPECT/CT had a sensitivity (93\%) similar to that $(99 \%)$ achieved by PET/CT with C-11-HED as a tracer (Franzius et al., 2006). A significant impact of SPECT/CT on therapeutic management was also demonstrated by Hillel et al., (2006) in neuroendocrine tumors. The addition of clinically relevant information for $40 \%$ of patients by SPECT/CT compared with SPECT was described by Gabriel et al., (2005). Co-registration with CT improves dose calculations in planning radionuclide therapy (Tang et al., 2001).

\subsubsection{Lymphoscintigraphy}

SPECT detects nodes missed on planar scintigraphy but stand-alone SPECT lacks anatomical landmarks. Pilot studies suggested incremental diagnostic efficacy in many 
cancers, including melanoma (Even-Sapir et al., 2003), head neck cancer (Wagner et al., 2007), cervical cancer (Zhang et al., 2006), invasive bladder cancer (Sherif et al., 2006), breast cancer (Gallowitsch et al., 2007) and prostate cancer (Vermeeren et al., 2009).

\subsubsection{Prostate cancer}

FDA approved the use of Prostascint (In-111 capromab-pendetide), a monoclonal antibodybased imaging agent for detecting prostate cancer in 1996 but it was soon realized that the low tumour/background ratio and the lack of anatomical information necessitates hybrid imaging (Terence et al., 2005). With the limited sensitivity of contrast enhanced CT in prostate cancer, the contribution lies mainly in the improvement of topographical orientation and mapping for planning of external radiation therapy.

\subsubsection{Dosimetry}

SPECT/CT might be useful for performing valid and clinically applicable dosimetry, for improving treatment planning, and for ensuring safe and effective radionuclide therapy (Boucek \& Turner 2005, Thierens et al., 2005, Prideaux et al., 2007, Lavely et al., 2007).

\subsection{Endocrinology}

\subsubsection{9m-Tc MIBI parathyroid adenoma}

CT co-registration is a valuable tool for the precise delineation of parathyroid adenomas with little incremental benefit from attenuation correction (Ruf et al., 2007) though others have seen little incremental benefit with non-diagnostic CT (Gayed et al., 2005).

\subsection{Cardiology}

SPECT attenuation correction with external sources was introduced in the early 1990s but, possibly due to financial and logistic problems got mixed reviews ever since (Dondi et al, 2004). Ten years later attenuation correction with CT was introduced (Masood et al., 2005) and the Society of Nuclear Medicine awarded its 2006 image of the year award to a cardiac SPECT/CT study (2006 Image of the year: focus on cardiac SPECT/CT. J Nucl Med. 2006; 47:14N-15N). Three different approaches were developed. The first was a low dose low resolution CT protocol aimed entirely at attenuation correction of SPECT data (Fricke et al., 2005), the second performed additional calcium scoring (Chang et al., 2009) and the third combined myocardial perfusion SPECT with a 64-slice CT angiography (CTA), initially only available on two different devices with digital fusion of the attenuation corrected SPECT and CTA (Gaemperli et al., 2007). Though feasible in principle and possibly identifying an independent risk, the value of assessing coronary calcifications or coronary morphology as part of a nuclear study needs to be validated in large prospective studies and appreciated in the light of the increased radiation exposure. At present attenuation correction in myocardial SPECT is especially useful in overweight patients and in women, however some degree of misregistration is still seen in the majority of the patients (Fricke et al., 2004, Goetze \& Wahl, 2007) and CT artefacts may be a problem. Recent developments aimed to reduce imaging times by using semiconductor detectors and a converging collimator to increase sensitivity and a 3D iterative reconstruction utilizing the CT-based attenuation map enabling SPECT acquisitions in typically 5 minutes (IQ•SPECT technology Siemens) (Corbet et al., 2010). 


\subsection{Neurology}

Until now no systematic analysis is available to assess differences between individually measured and conventionally calculated attenuation correction. Quantitative receptor imaging of fp-cit SPECT could be a perfect candidate for proving this principle.

\subsection{Bone scans}

Presently, SPECT/CT has added value in improved localization and characterization of bone lesions, both increasing sensitivity and specificity of bone scintigraphy. This was mainly achieved by identifying benign bone conditions with increased bone turnover (Utsunomiya et al., 2006). Until the introduction of SPECT/CT this has been routinely performed by comparing bone scanning with conventional (planar) X-ray and until now no additional benefit over the visual fusion of bone SPECT with planar X-ray, CT, or MRI studies has been confirmed (Horger et al., 2007). Thus, the "one-stop-shop" SPECT/CT has the drawback of increased radiation exposure. Low dose SPECT/CT may be a feasible compromise but may miss osteolytic lesions (Horger et al., 2004). "SPECT-guided CT" may be a better solution to the problem if it can be achieved logistically and is supported by the hybrid system (Roemer et al., 2006). Playing the devils advocate one may also assume that some of the lesions described as indeterminate in those scans could have been correctly identified by classical image features like pattern recognition (Puig et al., 1998) as it has been the clinical practice in times before hybrid imaging has hit the market.

\subsection{Infections}

Ga-67 scintigraphy, In-111 and Tc-99m labelled white blood cell scintigraphy is used to visualize infection. Physiological uptake and excretion and the lack of topographical information may hamper scan interpretation. SPECT/CT has been useful in the differentiation between bone and soft tissue infection (Fillippi et al., 2009).

\section{Unsolved challenges}

\subsection{Real integration in stead of two devices "glued" together}

Some of the early systems required two consoles to operate the system, one for the CT and one for the PET or SPECT. Some early PET/CTs incorporated a patient bore size that started at $70 \mathrm{~cm}$ for the CT and narrowed to about $59 \mathrm{~cm}$ for the PET system. In contrast to the statements of all manufacturers most commercially available systems are in essence still two separate machines (Figure 3).

The software of commercially available systems although combined under an apparently homogeneous surface still shows it different origins. Until the development of new detector types for both signals, movement in the interval between SPECT and CT data collection will continue to pose a problem.

\subsection{Imaging protocols}

A simple combination of traditional imaging protocols might not be the optimal use of hybrid imaging. Procedure guidelines and protocols are available for PET/CT (Boellaard et al., 2010) and SPECT/CT (Delbeke et al., 2006, Buck et al., 2008) but little efforts have been published so far to downsize conventional CT protocols if combined with Nuclear Medicine. The possible trade off of this has not been investigated, yet. Discussions have already begun about the reimbursement of diagnostic imaging as it is not apparent to most that a previous 


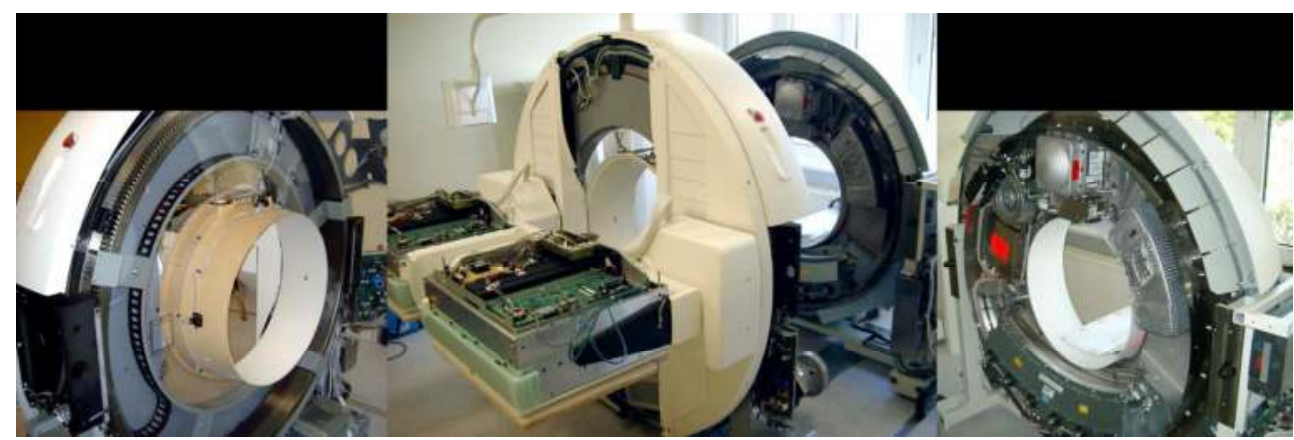

Fig. 3. Siemens Symbia T6: Left: Gantry of the dual head gamma camera, Center: both parts separated during service, Right: Gantry of the 6 slice MD CT

PET/CT study might not run other CT investigations obsolete. On the other hand, due to the higher number of CT investigations performed, many patients will already have undergone a diagnostic CT investigation before referral to their first Nuclear Medicine study and the duplicated CT further boosts the radiation burden. Current trends indicate that more and more centres use contrast enhancement and some regard the CT contrast agents and PET tracers as complementary (Antoch et al., 2004) but this may reflect common practice in radiology more than hard scientific evidence.

\subsection{Education and training, data reporting}

At present Nuclear Medicine and Radiology are different specialities in many countries with different training and licensing systems. Consequently the results of PET/CT and SPECT/CT require the competency and accreditation of individuals from both Nuclear Medicine and Radiology. Proposals for future training models have been put forward (White Paper, 2007) but first studies claim that only minimal additional training may be necessary to interpret both modalities (Narayanan et al., 2011). None of the commercially available RIS-NIS programmes is capable of simultaneous data reporting and validation by two different professionals. Standardization is mandatory as a recent survey (Cuocolo \& Breatnach, 2010) revealed a wide heterogeneity in the current practice of multimodality imaging in Europe.

\subsection{Diagnostic pathways}

All current clinical recommendations are based on the sequential use of different imaging modalities. If hybrid imaging should be used to its full diagnostic utility, we will have to rewrite conventional diagnostic paths as in many cases the optimized approach will start with hybrid imaging. However most of these pathways assume that the referrer has considerably narrowed the spectrum of possible differential diagnoses for a given problem, otherwise many conventional imaging steps will be necessary to narrow the problem to such an extent that a tailored approach is possible.

\subsection{Forensic liabilities}

The new hybrid devices produce a lot of additional information, not necessarily expected by the referrer. Data are accumulating that unexpected findings in PET/CT and SPECT/CT 
(Goetze \& Wahl 2007) as solitary pulmonary nodules, pneumonitis, and pleural effusion have clinical significance. The same problem has occurred in coronary CT protocols (Onuma et al., 2006, Schietinger et al., 2008).

\subsection{Improved PET/CT correction, quantitative imaging}

Whereas PET imaging was at least semi quantitative right from the start, quantitative SPECT has - in spite of early efforts (Rosenthal et al., 1995) - never reached prime time. The contribution of scatter, collimator response, depth dependent resolution and partial volume effect for accurate quantification have been determined for some investigations (Buvat et al., 2000), but only few data are available for the additional utility of CT for advanced data processing (Shcherbinin et al., 2008). On the other hand, contrast media and CT artefacts may severely hamper quantification of PET or SPECT (Sureshbabu \& Mawlawi, 2005).

\subsection{Costs}

It is often overlooked that the increased costs for hybrid imaging are not only due to equipment costs but also to room preparation and energy supply. In those institutions where only Nuclear Medicine or Radiology has been practiced in the past, structural radiation protection for the other modality has to be installed. CT has a higher energy demand than conventional gamma cameras and produces more heat, thus energy supply and air conditioning have to be adapted. Usually hybrid systems require larger space than conventional systems. Both, radiation protection and space requirements stress the statics of some buildings.

\section{Acknowledgement}

The project was in part supported by the "Medical Research Society Vienna D.C."

\section{References}

Abraham, T. \& Schöder, H. (2011). Thyroid cancer--indications and opportunities for positron emission tomography/computed tomography imaging. Semin Nucl Med, 41(2):121-38. 0001-2998

Almuhaideb, A., Papathanasiou, N., Bomanji, J. (2011). 18F-FDG PET/CT imaging in oncology. Ann Saudi Med, 31(1):3-13. 0975-4466

Ambrose, J. \& Hounsfield, G. (1973). Computerized transverse axial tomography. Br J Radiol, 46(542):148-9. 0007-1285

Antoch, G., Kanja, J., Bauer, S., et al. (2004). Comparison of PET, CT, and dual-modality PET/CT imaging for monitoring of imatinib (STI571) therapy in patients with gastrointestinal stromal tumors. J Nucl Med, 45:357-365. 0161-5505

Basu, S., Urhan, M., Rosenbaum, J., Alavi, A. (2011). PET and PET/CT in the Management of Thyroid Cancer. Meth Mol Biol, 727:205-24. 1064-3745.

Bauhs, JA., Vrieze, TJ., Primak, AN., Bruesewitz, MR., McCollough, CH. (2008). CT dosimetry: comparison of measurement techniques and devices. Radiographics, 28(1):245-53. 0271-5333 
BEIR VII (2006) Committee to Assess the Health Risks from Exposure to Low Levels of Ionizing Radiation, National Research Council. Health Risks From Exposure to Low Levels of Ionizing Radiation. Washington, DC: National Academies Press.

Beyer, T., Czernin, J., Freudenberg, LS. (2011). Variations in clinical PET/CT operations: results of an international survey of active PET/CT users. J Nucl Med, 52(2):303-10. 0161-5505

Beyer, T., Townsend, DW., Brun, T., et al. (2000). A combined PET/CT scanner for clinical oncology. J Nuc Med, 41:1369-1379. 0161-5 505

Boellaard, R., O'Doherty, MJ., Weber, WA., et al. (2010), FDG PET and PET/CT: EANM procedure guidelines for tumour PET imaging: version 1.0. Eur J Nucl Med Mol Imag, 37(1):181-200. 1619-7070

Boucek, JA. \& Turner, JH. (2005), Validation of prospective whole-body bone marrow dosimetry by SPECT/CT multimodality imaging in 131I-anti-CD20 rituximab radioimmunotherapy of non-Hodgkin's lymphoma. Eur J Nucl Med Mol Imag, 32:458-469. 1619-7070

Bruzzi, JF., Munden, RF., Truong, MT., et al. (2007), PET/CT of esophageal cancer: its role in clinical management. Radiographics, 27(6):1635-52. 0271-5333

Buck, AK., Nekolla, S., Ziegler, S., et al. (2008). SPECT/CT. J Nucl Med, 49(8):1305-19. 01615505

Burnham, C. \& Brownell, G., (1972). A multi-crystal positron camera. IEEE Trans Nucl Sci, 19:201-205, 0018-9499

Buvat, I., Soret, M., Hapdey, S., et al. (2000). Respective importance of scatter, collimator response, and partial volume effect corrections for accurate quantification 123I dopamine receptor imaging. IEEE Nucl Sci Symp Rec, 13:15-13/19

Büther, F., Stegger, L., Dawood, M., et al. (2007). Effective methods to correct contrast agentinduced errors in PET quantification in cardiac PET/CT. J Nucl Med, 48(7):1060-8. 0161-5505

Chang, SM. et al. (2009). The coronary artery calcium score and stress myocardial perfusion imaging provide independent and complementary prediction of cardiac risk. J Am Coll Cardiol, 54(20), 1872-82. 0735-1097

Chowdhury, FU. \& Scarsbrook, AF. (2008). The role of hybrid SPECT-CT in oncology: current and emerging clinical applications. Clin Radiol, 63(3):241-51. 0009-9260

Chowdhury, FU., Shah, N., Scarsbrook, AF., Bradley, KM. (2010). 18F FDG PET/CT imaging of colorectal cancer: a pictorial review. Postgrad Med J, 86(1013):174-82. 0032-5473

Coleman, RE., Delbeke, D., Guiberteau, MJ., et al. (2005). Joint Working Group of the American College of Radiology; Society of Nuclear Medicine; Society of Computed Body Tomography and Magnetic Resonance. Concurrent PET/CT with an integrated imaging system: intersociety dialogue from the Joint Working Group of the American College of Radiology, the Society of Nuclear Medicine, and the Society of Computed Body Tomography and Magnetic Resonance. J Am Coll Radiol, 2(7):568-84. 1546-1440

Cooper, KL., Harnan, S., Meng, Y., et al. (2011). Positron emission tomography (PET) for assessment of axillary lymph node status in early breast cancer: A systematic review and meta-analysis. Eur J Surg Oncol, 37(3):187-98. 0748-7983

Corbett, J. et al. (2010) Clinical Validation of Attenuation Corrected Cardiac Imaging with IQ-SPECT SPECT/CT. Abstract from the 2010 Society of Nuclear Medicine Meeting 
Cronin, CG., Swords, R., Truong, MT., et al. (2010). Clinical utility of PET/CT in lymphoma. AJR, 194(1):W91-W103. 1067-8654.

Cuocolo, A. \& Breatnach, E., (2010). Multimodality imaging in Europe: a survey by the European Association of Nuclear Medicine (EANM) and the European Society of Radiology (ESR). Eur J Nucl Med Mol Imag, 37(1):163-7. 1619-7070

Czernin, J., Benza, MR., Allen-Auerbacha, MS., (2010). PET/CT imaging: The incremental value of assessing the glucose metabolic phenotype and the structure of cancers in a single examination. Europ J Radiol, 73 (3) 470-480. 0720-048X

Delbeke, D., Coleman, RE., Guiberteau, MJ., et al., (2006). Society of Nuclear Medicine (SNM). Procedure Guideline for SPECT/CT Imaging 1.0. J Nucl Med, 47(7):1227-34. 0161-5505

Delbeke, D. \& Israel, O. (eds.), Hybrid PET/CT and SPECT/CT Imaging. Springer Science+Business Media 2010. Chapter I.1 History and Principles of Hybrid Imaging by JA Patton p. 28. ISSN 9780387928203.

Delbeke, D., Schöder, H., Martin, WH., Wahl, RL., (2009). Hybrid imaging (SPECT/CT and PET/CT): improving therapeutic decisions. Semin Nucl Med, 39(5):308-40. 0001-2998

Di Carli, MF., Dorbala, S., Meserve, J., et al., (2007), Clinical myocardial perfusion PET/CT. J Nucl Med, 48(5):783-93. 0161-5505

Dondi, M., Fagioli, G., Salgarello, M., Zoboli, S., et al., (2004). Myocardial SPECT: what do we gain from attenuation correction (and when)? Q J Nucl Med Mol Imag, 48:181187. 1824-4785

Engles, JM., Quarless, SA., Mambo, E., et al. (2006), Stunning and its effect on 3H-FDG uptake and key gene expression in breast cancer cells undergoing chemotherapy. $J$ Nucl Med, 47(4):603-8. 0161-5505

Eschmanna SM., Friedel, G., Paulsenc, F., et al., (2007). Repeat 18F-FDG PET for monitoring neoadjuvant chemotherapy in patients with stage III non-small cell. Lung cancer, 55 (2):165-171. 0169-5002

Even-Sapir, E., Keidar, Z., Sachs, J., et al. (2001). The New Technology of Combined Transmission and Emission Tomography in Evaluation of Endocrine Neoplasms. J Nucl Med, 42(7): 998-1004. 0161-5505

Even-Sapir, E., Lerman, H., Lievshitz, G., et al. (2003). Lymphoscintigraphy for sentinel node mapping using a hybrid SPECT/CT system. J Nucl Med, 44: 1413-1420. 0161-5505

Facey, K., Bradbury, I., Laking, G., Payne, E.. (2007). Overview of the clinical effectiveness of positron emission tomography imaging in selected cancers. Health Technol Assess, 11(44):iii-iv, xi-26. 1366-5278

Farid, K., Sibon, I., Fernandez, P., et al. (2009). Tc-99m HMPAO-SPECT with CT attenuation correction improves detection of epileptogenic areas. Clin Nucl Med, 34:290Y291. 0363-9762

Fillipi, L., Uccioli, L., Giurato, L., Schillaci, O., (2009). Diabetic Foot Infection: Usefulness of SPECT/CT for 99mTc-HMPAO-Labeled Leukocyte Imaging. JNM, 50(7), 1042-1046. 0161-5505

Filippi, V., Malamitsi, J., Vlachou, F., et al. (2011). The impact of FDG-PET/CT on the management of breast cancer patients with elevated tumor markers and negative or equivocal conventional imaging modalities. Nucl Med Comm, 32(2):85-90. 0143-3636

Fletcher, JW., Djulbegovic, B., Soares, HP., et al. (2008). Recommendations on the use of 18FFDG PET in oncology. J Nucl Med, 49(3):480-508. 0161-5505 
Franzius, C., Hermann, K., Weckesser, M., et al., (2006). Whole-body PET/CT with 11Cmetahydroxyephedrine in tumors of the sympathetic nervous system: feasibility study and comparison with 123I-MIBG SPECT/CT. J Nucl Med, 47:1635-1642. 0161-5505

Freudenberg, LS., Antoch, G., Schütt, P., et al. (2004). FDGPET/CT in re-staging of patients with lymphoma. Eur J Nucl Med Mol Imag, 31:325-329. 1619-7070

Fricke, H., Fricke, E., Weise, R., et al., (2004). A Method to Remove Artifacts in AttenuationCorrected Myocardial Perfusion SPECT Introduced by Misalignment Between Emission Scan and CT-Derived Attenuation Maps. J Nucl Med, 45(10), 1619-1625. 0161-5505

Fricke, E., Fricke, H, Weise R, et al. (2005). Attenuation Correction of Myocardial SPECT Perfusion Images with Low-Dose CT: Evaluation of the Method by Comparison with Perfusion PET. J Nucl Med, 46(5), 736-744. 0161-5505

Fulham, MJ., Carter, J., Baldey, A., et al., (2009). The impact of PET-CT in suspected recurrent ovarian cancer: A prospective multi-centre study as part of the Australian PET Data Collection Project. Gynecol Oncol, 112(3):462-8. 0090-8258

Gabriel, M., Hausler, F., Bale, R., et al., (2005). Image fusion analysis of 99mTcHYNICTyr(3)-octreotide SPECT and diagnostic CT using an immobilisation device with external markers in patients with endocrine tumours. Eur J Nucl Med Mol Imag, 32:1440-1451. 1619-7070

Gaemperli, O., Schepis, T., Valenta, I., et al., (2007). Cardiac Image Fusion from Stand-Alone SPECT and CT: Clinical Experience. J Nucl Med, 48:696-703. 0161-5505

Gallowitsch, HJ., Kraschl, P., Igerc, I., et al., (2007). Sentinel node SPECT-CT in breast cancer: can we expect any additional and clinically relevant information? Nucl Med, 46:252256. 0029-5566

Gambhir, SS., Hoh, CK., Phelps, ME., et al., (1996). Decision tree sensitivity analysis for costeffectiveness of FDG-PET in the staging and management of non-small-cell lung carcinoma. J Nucl Med, 37(9):1428-36. 0161-5505

Gámez, C., Rosell, R., Fernández, A., et al., (2006). PET/CT fusion scan in lung cancer: current recommendations and innovations. J Thorac Oncol, 1(1):74-7. 1556-0864

Gayed, IW., Kim, EE., Broussard, WF., et al., (2005). The value of 99mTc-sestamibi SPECT/CT over conventional SPECT in the evaluation of parathyroid adenomas or hyperplasia. J Nucl Med, 46:248-252. 0161-5505

Gearhart, SL., Frassica, D., Rosen, R., et al. (2006). Improved staging with pretreatment positron emission tomography/computed tomography in low rectal cancer. Ann Surg Oncol, 13: 397-404. 1068-9265

Goetze, S., Brown, TL., Lavely, WC., et al., (2007). Attenuation correction in myocardial perfusion SPECT/CT: effects of misregistration and value of reregistration. $\mathrm{J} \mathrm{Nucl}$ Med, 48:1090-1095. 0161-5505

Goetze, S. \& Wahl, RL. (2007). Prevalence of misregistration between SPECT and CT for attenuation-corrected myocardial perfusion SPECT. J Nuc Cardiol, 14, 200-206. 10713581

Groheux, D., Giacchetti, S., Espié, M., et al., (2011). Early monitoring of response to neoadjuvant chemotherapy in breast cancer with (18)F-FDG PET/CT: defining a clinical aim. Eur J Nucl Med Mol Imag, 38(3):419-25. 1619-7070 
Hasegawa, BH., Lang, TF., Brown, EL., et al., (1993). Object specific attenuation correction of SPECT with correlated dual-energy x-ray CT. IEEE Trans Nucl Sci, NS-40:12421252. 0018-9499

Heller, GV., Calnon, D., Dorbala, S,. (2009). Recent advances in cardiac PET and PET/CT myocardial perfusion imaging. J Nucl Cardiol, 16(6):962-9. 1071-3581

Hillel, PG., van Beek, EJ., Taylor, C., et al., (2006). The clinical impact of a combined gamma camera/CT imaging system on somatostatin receptor imaging of neuroendocrine tumours. Clin Radiol, 61:579-587. 0009-9260

Hoffmann, EJ., Phelps, ME., Mullani, NA., et al., (1976). Design and performance characteristics of a whole-body positron transaxial tomograph. J Nucl Med, 17(6):493-502. 0161-5505

Horger, M., Eschmann, SM., Pfannenberg, C., et al., (2004). Evaluation of Combined Transmission and Emission Tomography for Classification of Skeletal Lesions. Am J Roentgenol, 183, 655-661. 0361-803X

Horger, M. \& Bares, R. (2006). The role of single-photon emission computed tomography/computed tomography in benign and malignant bone disease. Semin Nucl Med, 36(4):286-94. 0001-2998

Horger, M., Eschmann, SM., Pfannenberg, C., et al., (2007). Added value of SPECT/CT in patients suspected of having bone infection: preliminary results. Arch Orthop Trauma Surg, 127(3):211-21. 0936-8051

Hutchings, M. \& Barrington, SF. (2009). PET/CT for therapy response assessment in lymphoma. J Nucl Med, 50 Suppl 1:21S-30S. 0161-5505

Hyde, NC., Prvulovich, E., Newman, L., et al., (2003). A new approach to pre-treatment assessment of the N0 neck in oral squamous cell carcinoma: the role of sentinel node biopsy and positron emission tomography. Oral Oncol, 39(4):350-60. 1368-8375

ICRP publication 105 (2007). Radiation protection in medicine. Ann ICRP; 37(6):1-63. 01466453

Jeong, HS., Baek, CH., Son, YI., et al., (2007). Use of integrated 18F-FDG PET/CT to improve the accuracy of initial cervical nodal evaluation in patients with head and neck squamous cell carcinoma. Head Neck, 29(3):203-10. 1043-3074

Kantorva, I., Lipska, L., Belohlavek, O., et al., (2003). PET preoperative staging of colorectal cancer comparison with conventional staging and its impact on treatment decision making. J Nucl Med, 44: 1784-8. 0161-5505

Kessler, ML. (2006). Image registration and data fusion in radiation therapy, Br J Rad, 79: 99$108,0007-1285$

Kim, TJ., Kim, HY., Lee, KW., Kim, MS. (2009). Multimodality assessment of esophageal cancer: preoperative staging and monitoring of response to therapy. Radiographics, 29(2):403-21. 0271-5333

Kinahan, PE., Hasegawa, BH., Beyer T. (2003). X-Ray-Based Attenuation Correction for Positron Emission Tomography/Computed Tomography Scanners. Sem Nucl Med, 33: 166-179. 0001-2998

Klabbers, BM., Lammertsma, AA., Slotman, BJ. (2003). The value of positron emission tomography for monitoring response to radiotherapy in head and neck cancer. Mol Imaging Biol, 5(4):257-70. 1536-1632

Kohlfürst, S., Igerc, I., Lobnig, M., et al., (2009). Posttherapeutic 131I SPECT-CT offers high diagnostic accuracy when the findings on conventional planar imaging are 
inconclusive and allows a tailored patient treatment regimen. Eur JNuc Med Mol Imag, 36(6), 886-893. 1619-7070

Kramer, EL. \& Noz, ME. (1991). CT/SPECT fusion for analysis of radiolabled antibodies: applications in gastrointestinal and lung cancinoma. Int J Rad Appl InstrumB, 18:2742. 0883-2897

Krishnasetty, V., Bonab, AA., Fischman, AJ., et al., (2008). Comparison of standard-dose vs low-dose attenuation correction $\mathrm{CT}$ on image quality and positron emission tomographic attenuation correction. J Am Coll Radiol, 5(4):579-84. 1546-1440

Krug, B., Crott, R., Roch, I., et al., (2010). Cost-effectiveness analysis of FDG PET-CT in the management of pulmonary metastases from malignant melanoma. Acta Oncol, 49(2):192-200. 0284-186X

Lang, TF., Hasegawa, BH., Liew, SC., et al., (1991). A prototype emission-transmission imaging system. IEEE Nucl Sci Symp Conf Rec, 3:1902-1906. 0018-9499

Leitha T. \& Staudenherz A. (2003). Frequency of diagnostic dilemmas in 131I whole body scanning. Nuc Med, 42(2):55-62. 0029-5566

Langer A. (2010). A systematic review of PET and PET/CT in oncology: a way to personalize cancer treatment in a cost-effective manner? BMC Health Serv Res, 10:283. 1472-6963

Lavely, WC., Goetze, S., Friedman, KP., et al., (2007). Comparison of SPECT/CT, SPECT, and planar imaging with single- and dual-phase $99 \mathrm{mTc}$-sestamibi parathyroid scintigraphy. J Nucl Med, 48:1084-1089. 0161-5505

Lejeune, C., Bismuth, MJ., Conroy, T., et al., (2005). Use of a decision analysis model to assess the cost-effectiveness of 18F-FDG PET in the management of metachronous liver metastases of colorectal cancer. J Nucl Med, 46(12):2020-2028. 0161-5505

MacManus, M., Nestle, U., Rosenzweig, KE., et al., (2009). Use of PET and PET/CT for radiation therapy planning: IAEA expert report 2006-2007. Radiother Oncol, 91(1):8594. 0167-8140

Mahmarian, JJ. (2007). Hybrid SPECT-CT: integration of CT coronary artery calcium scoring and angiography with myocardial perfusion. Curr Cardiol Rep, 9(2):129-35. 15233782

Mankoff DE. (2007). A Definition of Molecular Imaging. JNM; 48:18 -21N. 0161-5505

Masood, Y., Liu, YH., Depuey, G., et al. (2005). Clinical validation of SPECT attenuation correction using x-ray computed tomography-derived attenuation maps: multicenter clinical trial with angiographic correlation. J Nucl Cardiol, 12:676-686. 1071-3581. 1071-3581

Moore, KL., Palaniswaamy, G., White, B., et al., (2010). Fast, low-dose patient localization on TomoTherapy via topogram registration. Med Phys, 37(8):4068-77. 0094-2405

Munden, RF., Macapinlac, HA., Erasmus, JJ.. (2006). Esophageal cancer: the role of integrated CT-PET in initial staging and response assessment after preoperative therapy. J Thorac Imag, (2):137-45. 0883-5993

Nakamoto, Y., Senda, M., Okada, T., et al., (2008). Software-based fusion of PET and CT images for suspected recurrent lung cancer. Mol Imaging Biol, 10(3):147-53. 15361632

Narayanan D., Mdsen KS, Kalinyak JE., Berg WA. (2011). Interpretation of Positron Emission Mammography and MRI by Experienced Breast Imaging Radiologists: Performance and Observer Reproducibility, AJR, 196:971-981. 1067-8654 
Neuwirth, J., Hefner, A., Ernst G., Staudenherz A. (2010). Does decreasing of setting parameters of the $\mathrm{CT}$ at PET/CT devices minimize patient-dose without noteworthy degradation of attenuation correction? S02-07 Third European IRPA Congress, Helsinki, F Publisher: NSFS - Nordic Society for Radiation Protection

O'Daniel, JC., Stevens, DM., Cody, DD. (2005). Reducing radiation exposure from survey CT scans. AJR, 185(2):509-15. 1067-8654

Onuma, Y., Tanabe, K., Nakazawa, G., et al. (2006). Noncardiac findings in cardiac imaging with multidetector computed tomography. J Am Coll Cardiol, 48: 402-406. 0735-1097

Pan, T., Mawlawi, O., Nehmeh, SA., et al., (2005). Attenuation correction of PET images with respirators-averaged CT images in PET/CT. J Nuc Med, 46:1481-1487. 0161-5505

Patton JA. \& Turkington TG. (2008). SPECT/CT Physical Principles and Attenuation Correction. J Nuc Med Tech, 36 (1): 1-10. 0091-4916

Paulino, AC., Thorstad, WL., Fox T. (2003). Role of fusion in radiotherapy treatment planning. Semin Nucl Med, 33(3):238-43. 0001-2998

Pennant, M., Takwoingi, Y., Pennant, L., et al., (2010). A systematic review of positron emission tomography (PET) and positron emission tomography/computed tomography (PET/CT) for the diagnosis of breast cancer recurrence. Health Technol Assess, 14(50):1-103. 1366-5278

Perigauda, C., Bridjic, B., Roussela, JC., et al., (2009). Prospective preoperative mediastinal lymph node staging by integrated positron emission tomography-computerised tomography in patients with non-small-cell lung cancer. Eur J Cardio Thorac Surg, 36:731-736. 1010-7940

Pfannenberg, AC., Eschmann, SM., Horger, M., et al., (2003). Benefit of anatomicalfunctional image fusion in the diagnostic work-up of neuroendocrine neoplasms. Europ J Nuc Med Mol Imag, 30(6), 835-843. 1619-7070

Phelps, ME., Hoffman, EJ., Mullani, NA., Ter-Pogossian, MM., (1975). Application of annihilation coincidence detection to transaxial reconstruction tomography. J Nucl Med, 16(3):210-24. 0161-5505

Pichler, BJ., Wehrl, HF., Kolb, A., Judenhofer, MS. (2008). Positron emission tomography/magnetic resonance imaging: the next generation of multimodality imaging? Semin Nucl Med, 38(3):199-208. 0001-2998

Prideaux, AR., Song, H., Hobbs, RF., et al., (2007). Three-dimensional radiobiologic dosimetry: application of radiobiologic modeling to patient-specific 3-dimensional imaging-based internal dosimetry. J Nucl Med, 48:1008-1016. 0161-5505

Puig, S., Staudenherz, A., Steiner B, et al., (1998). Differential diagnosis of atypically located single or double hot spots in whole bone scanning. J Nucl Med, 39(7):1263-6. 01615505

Reinartz, P., Wieres, FJ., Schneider, W., et al., (2004). Side-by-side reading of PET and CT scans in oncology: which patients might profit from integrated PET/CT? Eur J Nucl Med Mol Imag, 31:1456-1461. 1619-7070

Römer, W., Nömayr, A., Uder, M., et al., (2006). SPECT-Guided CT for Evaluating Foci of Increased Bone Metabolism Classified as Indeterminate on SPECT in Cancer Patients. J Nuc Med, 47(7), 1102-1106. 0161-5505

Rosenthal, MS., Cullom, J., Hawkins, W., et al., (1995). Quantitative SPECT Imaging: A Review and Recommendations by the Focus Committee of the Society of Nuclear 
Medicine Computer and Instrumentation Council. J Nucl Med, 36:1489-1513. 01615505

Ruf, J., Seehofer, D., Denecke, T., et al. (2007). Impact of image fusion and attenuation correction by SPECT-CT on the scintigraphic detection of parathyroid adenomas. Nuc Med, 46:15-21. 0029-5566

Schietinger, BJ., Bozlar, U., Hagspiel, KD., et al. (2008). The prevalence of extracardiac findings by multidetector computed tomography before atrial fibrillation ablation. Am Heart J, 155:254-259. 0002-8703

Schillaci, O. (2005). Hybrid SPECT/CT: a new era for SPECT imaging? Eur J Nucl Med Mol Imag, 32:521-524. 1619-7070

Schöder, H., Yeung, HW., Gonen, M., et al., (2004). Head and neck cancer: clinical usefulness and accuracy of PET/CT image fusion. Radiology, 231(1):65-72. 0033-8419

Schwaiger, M., Ziegler, SI., Nekolla, SG. (2010). PET/CT challenge for the non-invasive diagnosis of coronary artery disease. Eur J Radiol, 73(3):494-503. 0720-048X

Shcherbinin, S., Celler, A., Belhocine, T., et al., (2008). Accuracy of quantitative reconstructions in SPECT/CT imaging. Phys Med Biol, 53: 4595-4604. 0031-9155

Segaert, I., Mottaghy, F., Ceyssens, S., et al., (2010). Additional value of PET-CT in staging of clinical stage IIB and III breast cancer. Breast J, 16(6):617-24. 1075-122X

Sherif, A., Garske, U., de la Torre, M., Thorn, M. (2006). Hybrid SPECT-CT: an additional technique for sentinel node detection of patients with invasive bladder cancer. Eur Urol, 50:83-91.

Slomka PJ. (2004). Software Approach to Merging Molecular with Anatomic Information J Nucl Med, 45:36S-45S. 0161-5505

Stabin, M., Stubbs, JB., Toohey, RE. (1996). Radiation Dose Estimates for Radiopharmaceuticals. Oak Ridge, TN: Radiation Internal Dose Information Center, ORNL

Stemberger, A., Leitha, T., Staudenherz, A. (2011). Diagnostic reference value. Critical evaluation of the term with the example of nuclear medicine studies in Austria. NucMed, Feb 21;50(2). [Epub ahead of print]

Strobel, K., Dummer, R., Husarik, DB., et al., (2007). High-risk melanoma: accuracy of FDG PET/CT with added CT morphologic information for detection of metastases. Radiology, 244(2):566-74. 0033-8419

Sureshbabu, W., Mawlawi, O., (2005). PET/CT Imaging Artifacts, J Nuc Med Tech, 33 (3): 156-161. 0091-4916

Tang, HR., Da Silva, AJ., Matthay, KK., et al., (2001). Neuroblastoma Imaging Using a Combined CT Scanner-Scintillation Camera and 131I-MIBG. J Nucl Med, 42(2), 237247. 0161-5505

Terence, Z., Wong, TZ., Turkington, TG., et al., (2005). ProstaScint (Capromab Pendetide) Imaging Using Hybrid Gamma Camera-CT Technology. AJR, 184(2):676-80. 10678654

Ter-Pogossian, MM., Phelps, ME., Hoffman, EJ., Mullani, NA. (1975). A positron-emission transaxial tomograph for nuclear imaging (PET). Radiology, 114(1):89-98. 0033-8419

Thierens HM, Monsieurs MA, Bacher K. (2005). Patient dosimetry in radionuclide therapy: the whys and the wherefores. Nucl Med Comm, 26:593-599.

Townsend, DW., Beyer, T., Kinahan, PE., et al., (1998). The SMART scanner: A combined PET/CT tomography for clinical oncology. IEEE Nucl Sci Symp Conf Rec, 2:11701174, paper M5-1. 0018-9499 
Utsunomiya, D., Shiraishi, S., Imuta, M., et al., (2006). Added value of SPECT/CT fusion in assessing suspected bone metastasis: comparison with scintigraphy alone and nonfused scintigraphy and CT. Radiology, 238(1):264-71. 0033-8419

Veena, R., Iyer, VR., Lee, SI. (2010). MRI, CT, and PET/CT for Ovarian Cancer Detection and Adnexal Lesion Characterization. AJR, 194:311-321. 1067-8654

Vermeeren, L., Valdés Olmos, RA., Meinhardt, W., et al., (2009). Value of SPECT/CT for dectection and anatomic localization of sentinal lymph nodes before laparascopic sentinel node lymphadenectomy in prostate cardinoma. J Nuc Med, 50(6), 865-870. 0161-5505

von Schulthess, GK. \& Pelc, NJ. (2002). Integrated-modality imaging: the best of both worlds. Acad Radiol, 9:1241-4. 1076-6332

Wagner, A., Kermer, C., Zettinig, G., et al., (2007). Validity of sentinel lymph node (SLN) detection following adjuvant radiochemotherapy (RCT) in head and neck squamous cell carcinoma (HNSCC). Technol Cancer Res Treat, 6(6):655-60. 1533-0346

Wahl, RL., Jacene, H., Kasamon, Y., Lodge, MA. (2009). From RECIST to PERCIST: Evolving Considerations for PET response criteria in solid tumors. J Nucl Med, 50 Suppl 1:122S-50S. 0161-5505

Walker, AJ., Spier, BJ., Perlman, SB., et al., (2011). Integrated PET/CT fusion imaging and endoscopic ultrasound in the pre-operative staging and evaluation of esophageal cancer. Mol Imag Biol, 13(1):166-71. 1536-1632

White Paper:

http://www.spmn.org/documentos/2007_FINAL_White_Paper_Cuocolo_Adam_ 23_05_07.pdf

Wiering, B., Vogel, WV., Ruers, TJ., Oyen WJ. (2008). Controversies in the management of colorectal liver metastases: role of PET and PET/CT. Dig Surg, 25(6):413-20. 02534886

Weber, WA. \& Figlin, R. (2007). Monitoring Cancer Treatment with PET/CT: Does it make a difference. J Nucl Med, 48:36S-44S. 0161-5505

Weber, WA., Ott, K., Becker, K., et al., (2001). Prediction of response to preoperative chemotherapy in adenocarcinomas of the esophagogastric junction by metabolic imaging. J Clin Oncol, 19(12):3058-65. 0732-183X

Yamamoto, Y., Nishiyama, Y., Monden, T., et al., (2003). Clinical usefulness of fusion of 131I SPECT and CT images in patients with differentiated thyroid carcinoma. J Nucl Med, 44:1905-1910. 0161-5505

Yang, DH., Min, JJ., Song, HC., et al., (2011). Prognostic significance of interim (18)F-FDG PET/CT after three or four cycles of R-CHOP chemotherapy in the treatment of diffuse large B-cell lymphoma. Eur J Cancer, Feb 17. [Epub ahead of print]

Yen, RF., Yen, MF., Hong, RL., et al., (2009). The Cost-utility Analysis of 18-Fluoro-2Deoxyglucose Positron Emission Tomography in the Diagnosis of Recurrent Nasopharyngeal Carcinoma. Acad Radiol, 16(1):54-60. 1076-6332

Zhang, WJ., Zheng, R., Wu, LY., et al., (2006). Clinical application of sentinel lymph node detection to early stage cervical cancer [in Chinese]. Chin J Canc/Ai Zheng, 25:224228. 1000-467X 


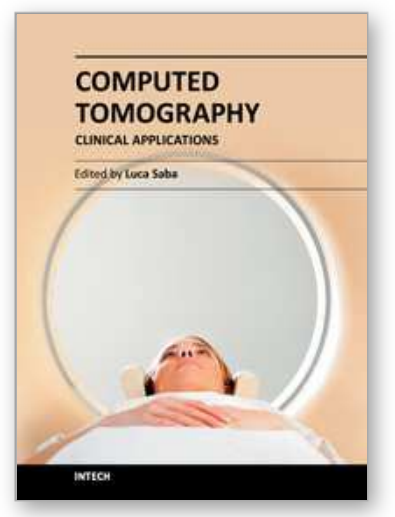

\author{
Computed Tomography - Clinical Applications \\ Edited by Dr. Luca Saba
}

ISBN 978-953-307-378-1

Hard cover, 342 pages

Publisher InTech

Published online 05, January, 2012

Published in print edition January, 2012

Computed Tomography (CT), and in particular multi-detector-row computed tomography (MDCT), is a powerful non-invasive imaging tool with a number of advantages over the others non- invasive imaging techniques. CT has evolved into an indispensable imaging method in clinical routine. It was the first method to non-invasively acquire images of the inside of the human body that were not biased by superimposition of distinct anatomical structures. The first generation of CT scanners developed in the 1970 s and numerous innovations have improved the utility and application field of the $\mathrm{CT}$, such as the introduction of helical systems that allowed the development of the "volumetric CT" concept. In this book we want to explore the applications of CT from medical imaging to other fields like physics, archeology and computer aided diagnosis. Recently interesting technical, anthropomorphic, forensic and archeological as well as paleontological applications of computed tomography have been developed. These applications further strengthen the method as a generic diagnostic tool for non- destructive material testing and three-dimensional visualization beyond its medical use.

\title{
How to reference
}

In order to correctly reference this scholarly work, feel free to copy and paste the following:

Thomas Leitha and Anton Staudenherz (2012). Hybrid PET/CT and SPECT/CT Imaging, Computed Tomography - Clinical Applications, Dr. Luca Saba (Ed.), ISBN: 978-953-307-378-1, InTech, Available from: http://www.intechopen.com/books/computed-tomography-clinical-applications/hybrid-pet-ct-and-spect-ctimaging

\section{INTECH}

open science | open minds

\section{InTech Europe}

University Campus STeP Ri

Slavka Krautzeka 83/A

51000 Rijeka, Croatia

Phone: +385 (51) 770447

Fax: +385 (51) 686166

www.intechopen.com

\section{InTech China}

Unit 405, Office Block, Hotel Equatorial Shanghai

No.65, Yan An Road (West), Shanghai, 200040, China

中国上海市延安西路 65 号上海国际贵都大饭店办公楼 405 单元

Phone: +86-21-62489820

Fax: $+86-21-62489821$ 
(C) 2012 The Author(s). Licensee IntechOpen. This is an open access article distributed under the terms of the Creative Commons Attribution 3.0 License, which permits unrestricted use, distribution, and reproduction in any medium, provided the original work is properly cited. 